\title{
Análisis crítico de guía clínica GES gran quemado versión 2007*
}

\author{
Drs. STEFAN DANILLA E. ${ }^{1,2}$, HERNÁN NORAMBUENA B. ${ }^{4}$,SUSANA SEARLE F. ${ }^{3}$, \\ PATRICIO FUENTES F. ${ }^{2}$, PAULO CASTILLO D. ${ }^{2}$, MARCELO FERES W. ${ }^{2}$, ANTONIO TRONCOSO R. ${ }^{2}$
}

1 Unidad de Cirugía Plástica, Departamento de Cirugía, Hospital Clínico Universidad de Chile .

2 Servicio de Cirugía Plástica, Hospital de Carabineros.

3 Departamento de Cirugía Pontificia Universidad Católica de Chile.

4 Servicio de Cirugía Plástica, Hospital Dirección de Previsión de Carabineros de Chile (DIPRECA). Santiago, Chile.

\begin{abstract}
Critical appraisal of clinical guideline

Objective: Critical appraisal of the clinical practice guideline (CPG) "Gran Quemado-Extensive Burn Patients"(2007 issue) corresponding to "Garantías Explícitas en Salud- Explicit Health Guarantees" (GES). Material and Methods: The CPG was evaluated using the previously validated AGREE instrument. This instrument evaluates a series of items organized in 6 domains, that capture different dimensions of the guidelines quality comparing the scores obtained with a maximum theoretical score. The $\mathrm{CPG}$ was evaluated by three independent and masked authors applying the AGREE instrument. Results: Stratified by domain, in the "scope and purpose" domain there was an $88.9 \%$ of compliance; in "stakeholder involvement" $47.9 \%$; in the "rigour of development" $47.6 \%$; in "clarity and presentation" $79.2 \%$; in "applicability" $30.6 \%$ and $75 \%$ in the "editorial independence" domain; reaching a $44.9 \%$ final score of compliance. Conclusions: The score obtained was below $50 \%$ of the optimum for a CPG. The detailed analysis by domain makes evident the areas that may be subject of improvement, so as to optimize the applicability of the CPG and therefore guarantee better health care and treatment results for all burn patients benefiting from the "Explicit Health Guarantees".

Key words: Critical appraisal, practice guidelines, quality assessment, evidence based medicine, AGREE, burn.
\end{abstract}

\section{Resumen}

Objetivo: Evaluar críticamente la guía de práctica clínica (GPC) de Gran Quemado correspondiente a las Garantías Explícitas en Salud (GES) versión 2007. Material y Método: La evaluación se realizó con el instrumento AGREE el cual ha sido previamente validado. El instrumento AGREE evalúa una serie de ítems en 6 dominios entregando un puntaje específico que se compara con un máximo teórico. Tres autores

*Recibido el 20 de abril de 2010 y aceptado para publicación el 27 de julio de 2010.

Este trabajo fue previamente presentado en: "V Congreso Chileno de Quemaduras", 15-17 octubre 2008, Santiago de Chile" y "VII Jornadas Chilenas de Cirugía Plástica", noviembre de 2009, Coquimbo, Chile.

Correspondencia: Dr. Stefan Danilla E. Félix de Amesti 94 Dpto. 73, Santiago, Chile. drstefandanilla@gmail.com 
en forma independiente y enmascarada evaluaron la GPC y puntuaron de acuerdo al instrumento utilizado. Resultados: Estratificando por dominio, en "alcance y objetivo" se obtuvo un 88,9\% de cumplimiento; en participación de los implicados 47,9\%; en rigor en la elaboración 47,6\%; en claridad y presentación 79,2\%; en aplicabilidad 30,6\% y 75\% en independencia editorial; entregando un puntaje final de 44,9\% de cumplimiento. Conclusiones: El puntaje obtenido fue menor al 50\% del óptimo para una GPC. El análisis detallado por dominio entrega en forma detallada las áreas susceptibles de perfeccionar para optimizar la aplicabilidad de la guía clínica y de tal forma garantizar la mejoría en el cuidado y los resultados del tratamiento de los pacientes quemados beneficiarios de las Garantías Explícitas en Salud.

Palabras clave: Análisis crítico, guía clínica, medicina basada en la evidencia, AGREE, quemados, quemaduras.

En la década de los 90, varios países de América Latina y el Caribe iniciaron procesos de reforma social con énfasis en el sector salud. Chile, en el año 2000 , inicia su proceso enviando al congreso un proyecto de ley que busca regular los derechos y deberes de los pacientes, otorgar protección financiera a los individuos y mejorar la gestión y administración de los prestadores de salud públicos y privados ${ }^{1}$.

Uno de los pilares de la reforma de salud chilena fue el denominado Plan AUGE (Acceso Universal con Garantías Explícitas) el cual busca normar y estandarizar el acceso y cuidado en una serie de patologías seleccionadas en base a la carga de enfermedad que genera al país. Estos se refieren al derecho que otorga la Ley a todos los afiliados al sistema público (Fondo Nacional de Salud, FONASA) o privado (Instituciones de Salud Previsional, ISAPRE), y a sus respectivas cargas, para ser atendido en condiciones especiales que garantizan su acceso, calidad, oportunidad y protección financiera, en caso de sufrir cualquiera de los problemas de salud definidos por el Ministerio de Salud².

Una de las herramientas en las que este plan se apoya, son las Guías GES (Garantía Explícita en Salud), las cuales se estructuran como guía de práctica clínica (GPC) que contiene las intervenciones preventivas, diagnósticas, terapéuticas o paliativas recomendadas en base a la mejor evidencia disponible para cada patología. Estas recomendaciones, se transforman en parte de las garantías explícitas que los pacientes están en derecho de exigir y por otra parte, las instituciones de la red AUGE en la obligación de otorgar ${ }^{2}$.

Las quemaduras, son un problema de salud a nivel mundial, que afectan principalmente a países en vías de desarrollo ${ }^{3}$, Chile no es la excepción, donde las quemaduras provocan una carga de enfermedad importante, generando un gran impacto social y económico tanto para el individuo que sufre la lesión, como para su familia, entorno social y sistema de salud $^{4}$. En el año 2007 se incluyó el Gran Quemado como una de las patologías cubiertas por el sistema GES, publicándose en julio de 2007 la guía clínica para su manejo ${ }^{5}$.
Las Guías de Práctica Clínica, han surgido como una serie de recomendaciones desarrolladas de forma sistemática para ayudar al profesional de la salud y al paciente a tomar las decisiones adecuadas en circunstancias clínicas específicas ${ }^{6}$. Así, elabora recomendaciones explícitas con la intención definida de influir en la práctica de los clínicos ${ }^{7}$.

La aparición de múltiples GPC de calidad y aplicabilidad dudosa, llevó al desarrollo de instrumentos especializados para determinar la calidad metodológica de éstas, entendiéndose como calidad, la confianza en que los sesgos potenciales del desarrollo de la guía han sido detectado en forma adecuada y en que las recomendaciones son válidas interna y externamente, así como también la factibilidad de su aplicación en la práctica clínica ${ }^{6}$. Ya en la década de los noventa, con la introducción del concepto de Medicina Basada en la Evidencia (MBE) ${ }^{8}$, hubo un cambio en la formulación de las guías, evolucionando de consensos de profesionales y especialistas a recomendaciones basadas en la mejor evidencia científica disponible, cambiando de esta forma las exigencias en su calidad. Chile no ha sido ajeno a este fenómeno, generándose diversos grupos de práctica y enseñanza de la MBE y de la Epidemiología Clínica, destacando especialmente en cirugía el grupo de CIGES de la Universidad de la Frontera ${ }^{9,10}$.

Antes de implementar una guía clínica, es necesario asegurar su validez, reproducibilidad y confiabilidad, de tal forma que al implementarse se logren los objetivos establecidos y que todos los usuarios de la guía interpretarán las recomendaciones de la misma forma ${ }^{11}$.

Para lo anterior, surgió la necesidad de crear un instrumento común, válido y transparente tanto para el buen desarrollo de las guías, como para la evaluación de su validez y aplicabilidad. Con ese fin, en 1998, se desarrolla el instrumento AGREE (Appraisal of Guidelines Research \& Evaluation $)^{12-17}$. Este consistió en un grupo de colaboración internacional de investigadores, que tuvieron como objetivo mejorar la calidad y eficiencia de las GPC, evaluando tanto la calidad de la información aportada en el documento, como también la calidad de los 
aspectos recomendados. Ofreciendo una valoración de la validez de la GPC, la probabilidad de que la guía efectivamente logre el objetivo y los resultados esperados es mayor ${ }^{13}$.

En concreto, el AGREE consiste en una evaluación de 23 ítems que se agrupan en 6 dominios: 1) Alcance y objetivo; 2) Participación de los implicados; 3) Rigor en la elaboración; 4) Claridad y presentación; 5) Aplicabilidad; 6) Independencia editorial $^{13,18}$.

\section{Objetivo}

Nuestro objetivo fue analizar críticamente la guía de práctica clínica GES Gran Quemado versión 2007, utilizando el instrumento AGREE, para realizar un diagnóstico de la calidad actual de ésta y efectuar recomendaciones para su mejoría en la versión siguiente.

\section{Material y Método}

Se realizó una revisión independiente y enmascarada de la GPC AUGE Gran Quemado por 3 profesionales con instrucción formal en Medicina Basada en Evidencia, aplicando el instrumento AGREE.

Las 23 preguntas (ítems) que conforman las 6 áreas claves (Dominios) se graduaron mediante una escala ordinal Likert como se ejemplifica en la Tabla 1 (el instrumento completo puede observarse en el anexo 1).

Luego se calculó el puntaje estandarizado por dominio, el cual entrega el porcentaje de puntaje alcanzado por la evaluación con respecto al máximo teórico, es decir, el porcentaje alcanzado refleja la calidad de la GPC con respecto a una GPC ideal (Figura 1).

\section{Control de Sesgos}

Para optimizar la confiabilidad del análisis, se seleccionó a profesionales con experticia clínica y conocimiento formal en Epidemiología Clínica y MBE. Además, se calculó el grado de acuerdo (índice Kappa) entre los evaluadores y si existió diferencia entre las evaluaciones de cada uno (Kruskall-Wallis). La estabilidad interna del instrumento se graduó con el alfa de Cronbach. Todas las pruebas de hipótesis se realizaron con un nivel de error tipo I del 5\% (Stata 9.2 ®, Stata Corp, TX, USA).

\section{Resultados}

La concordancia entre los observadores fue $85,99 \%(\kappa=0,5293, \mathrm{p}=0,0025)$. Así mismo se obtuvo una estabilidad interna de 95,67\%. No existió diferencia significativa entre los puntajes obtenidos entre evaluadores $(p=0,3679)$. El puntaje final obtenido por la guía fue de $44,9 \%$. Al evaluar en forma independiente los dominios que integran el instrumento de evaluación se observan los resultados desglosados en la Tabla 2.

A continuación se describe el análisis por dominio.

Tabla 1. Ejemplo de ítems del instrumento AGREE

\begin{tabular}{|c|c|c|c|c|c|}
\hline Muy de acuerdo & 4 & 3 & 2 & 1 & Muy en desacuerdo \\
\hline \multicolumn{6}{|c|}{ Los criterios para seleccionar la evidencia se describen con claridad } \\
\hline Muy de acuerdo & 4 & 3 & 2 & 1 & Muy en desacuerdo \\
\hline
\end{tabular}

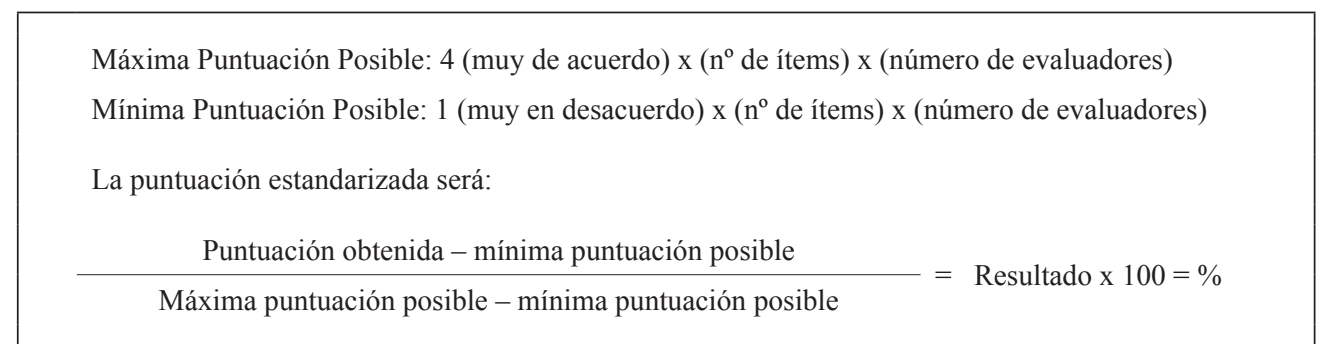

Figura 1. La puntuación estandarizada entrega una relación porcentual del puntaje alcanzado por la guía de práctica clínica evaluada con respecto al máximo teórico posible. 
Tabla 2. Resultados del Análisis por Dominio de AGREE

\begin{tabular}{lc}
\hline Dominio & $\mathbf{\%}$ \\
\hline Alcance y objetivo & 88,9 \\
Participación de los implicados & 47,9 \\
Rigor en la elaboración & 47,6 \\
Claridad y presentación & 79,2 \\
Aplicabilidad & 30,6 \\
Independencia editorial & 75,0 \\
\hline
\end{tabular}

Alcance y objetivo (88,9\%): En este dominio se evalúa la claridad de descripción de los objetivos, de los aspectos clínicos cubiertos y los pacientes a quienes se pretende aplicar la guía. Si bien los objetivos de la GPC y los aspectos clínicos cubiertos se encuentran claramente descritos, el grupo de pacientes a los cuales se pretende aplicar está poco definido. Específicamente se señala que tienen acceso AUGE todos los pacientes con Índice de Gravedad de Garcés (IGG) mayor a 70 puntos, lo que implica que todo paciente mayor a 70 años, aunque tenga una quemadura epidérmica del $1 \%$ de la superficie corporal total, de acuerdo a la GPC sería beneficiario AUGE. Se señala además que son beneficiarios los quemados con "Patologías graves", lo cual también, al ser subjetivo limita la aplicabilidad de la GPC.

Participación de los implicados (47,9\%): Este grupo se refiere al grado en el que la guía representa el punto de vista de todos los profesionales implicados en el cuidado de los pacientes a los cuales está dirigida la GPC. El grupo que desarrolló la GPC incluyó profesionales de varios rubros relacionados con el tratamiento integral del quemado: cirugía plástica, cirugía infantil, UCI adulto/pediátrico, kinesiólogos, terapeutas ocupacionales, pediatras y anestesiólogo; sin embargo, existen profesionales de vital importancia en el manejo interdisciplinario del paciente quemado que no participaron de la confección de la GPC, destacando: enfermería, psiquiatría, psicología, ingeniero/médico administrativo y asistente social. Tampoco se tuvo en cuenta los puntos de vista del paciente y sus preferencias respecto al tratamiento, lo cual por ejemplo ha generado conflictos en la aplicación de la GPC en provincias, donde el paciente tiene que ser trasladado a Santiago, con el menoscabo al entorno social del paciente, especialmente el pediátrico, ya que los costos de traslado de su familia no están cubiertos por el AUGE. Los usuarios diana de esta guía están descritos en forma muy global, genérica; no hay una definición específica de los profesionales a los que va dirigido, además, la GPC no fue aplicada ni probada entre los usuarios diana antes de su publicación.

Rigor en la elaboración (47,6\%): Los métodos de obtención de la evidencia para la redacción de la guía, formular las recomendaciones y actualizarlas están escasamente descritos. Existe una referencia breve acerca de la forma en que se realizó la revisión de la evidencia, sin metodología clara respecto a su obtención, tampoco está claro quien la realizó refiriéndose sólo a "un experto externo al ministerio". No se describen los criterios utilizados en la búsqueda ni en la selección de la evidencia disponible. Además, carece de relación explícita entre las recomendaciones y la evidencia en las que se basan, es decir, no existe una referencia asociada a cada una de las recomendaciones de la GPC. Si bien existe una síntesis de la evidencia en un anexo, no se señala el grado de recomendación asociado a cada uno, ni el análisis crítico individual de los estudios más importantes utilizados para formular las recomendaciones. La metodología empleada para formular las recomendaciones se encuentra definida en la guía. Se encuentran descritos de manera simple los beneficios de salud, efectos secundarios y riesgos pero no se utilizan indicadores específicos de medidas de asociación como riesgo relativo (RR), número necesario a tratar (NNT), likelihood ratio (LR), tasa de daño/beneficio u otras medidas de certeza, precisión, seguridad o eficacia que permitan evaluar la magnitud de la aplicación de las distintas intervenciones y la relevancia de las recomendaciones. Con la información entregada, no queda claro si esta guía fue revisada por expertos antes de su publicación. En cuanto a la actualización de la GPC, sólo hay una referencia a que se realizará cada dos años, sin embargo, no especifica ni se presenta un programa ni estrategia específicos para su actualización.

Claridad y presentación (79,2\%): Las recomendaciones son específicas y no son ambiguas, presentándose claramente las distintas opciones para el manejo de la enfermedad entregadas como diagramas de flujo y esquemas anexos al texto. También, las recomendaciones clave son claramente identificables en el texto y la GPC se apoya en herramientas útiles para su aplicación; por lo que el presente dominio fue uno de los con mayor puntuación en la evaluación de la GPC.

Aplicabilidad (30,6\%): Este punto hace referencia a las posibles consecuencias en recursos humanos, físicos, organizacionales o económicos de la aplicación de la GPC. En este aspecto la GPC presenta importantes deficiencias respecto a las barreras de gestión, administración de recursos, análisis de 
costos y beneficios. No se identifican barreras organizativas a la hora de aplicar las recomendaciones, los costos asociados a su implementación o métodos para monitorizar la aplicación y utilidad de la GPC para realizar auditorías.

Independencia editorial (75,0\%): Se declara explícitamente que no existen conflictos de interés respecto a los temas abordados en la GPC. Sin embargo, la declaración no queda clara si se refiere a los productos farmacéuticos recomendados o a la fuente de financiamiento. El hecho de que la mayoría de los autores trabajen afiliados al sistema público y la GPC haya sido financiada por el estado, sin que haya sido revisada por un comité externo de expertos, pudiese ser interpretado como conflicto de interés.

\section{Discusión}

\section{Calidad de las guías clínicas}

Cuando las GPC se analizan bajo el rigor del análisis crítico, destaca en general que son subóptimas respecto a los estándares metodológicos ${ }^{12,15,18,19}$. Shaneyfelt et $\mathrm{al}^{17}$, estudió la validez de las GPC basado en el cumplimiento de estándares metodológicos adecuados en su desarrollo, al analizar los principios metodológicos de 279 guías entre los años 1985 y 1997, sólo 43,1\% cumplían con éstos: 51,1\% cumplía con el formato de desarrollo de la guía, en $33,6 \%$ se identificó y resumió la evidencia y en $46 \%$ hubo formulación adecuada de las recomendaciones. La adherencia a los estándares de desarrollo mejoró desde 1985 a 1997 (36,9\% a 50,4\% respectivamente, $\mathrm{p}<0,001)$, sin embargo, el ítem identificación de la evidencia clínica no mostró mejoría en el tiempo, siendo siempre deficiente $(36,6 \%$ previo al año 1990 , y 36,1\% después del año 1995). No hubo diferencia en la calidad de las GPC según el origen de la guía (subespecialistas, sociedades, gobierno, etc.). Grilli et al ${ }^{15}$, analizó la calidad de $431 \mathrm{GPC}$, basado en el reporte de los profesionales involucrados en su desarrollo, estrategia utilizada para la recolección de la evidencia y clasificación de las recomendaciones según la calidad de su evidencia, arrojando que sólo el 5\% de las GPC cumplieron con los tres criterios de calidad.

\section{Impacto de las GPC}

Como nunca, ha habido un interés creciente acerca del uso e implementación de GPC, ya que suponen una disminución en las prácticas inadecuadas y consiguiente mejoría de los cuidados en salud. Al evaluar el impacto de las GPC en la práctica médica, traducido en mejoría de resultados clínicos y capacidad para cambiar el comportamiento de los profesionales de la salud, ha sido publicado que las GPC que cumplían con una metodología científica rigurosa, mejoraron significativamente los resultados clínicos luego de su implementación en un 93\% de los $\operatorname{casos}^{6}$. Estos resultados alentadores se mantuvieron, en la publicación por el grupo de Grimshaw $^{16,20}$. En un trabajo posterior, al analizar $91 \mathrm{GPC}$, se describe que en 87 estudios donde se evaluaba el efecto en procesos de salud, medido como adherencia a las recomendaciones, 81 mostraban clara mejoría, al igual que 12/17 en donde hubo cambios significativos en los resultados evaluados. Estos trabajos sugieren fuertemente que las GPC adecuadamente desarrolladas pueden cambiar la práctica médica y mejorar los resultados en el cuidado de los pacientes. Sin embargo, esta afirmación es aún controversial. En una revisión sistemática de Worrall et $\mathrm{al}^{21}$, con el objetivo de evaluar la efectividad de las GPC a nivel de la salud primaria, concluye que los estudios eran poco sensibles para determinar mejoría en los resultados, quedando pendiente la necesidad de mejores trabajos para demostrar que la implementación de las guías efectivamente tiene un impacto en la mejoría del cuidado de los pacientes.

\section{Importancia de recomendaciones $\mathrm{MBE}$}

Múltiples estudios demuestran que hay una tendencia a reconocer que los resultados tanto para los pacientes, el personal de salud y organizaciones de salud, mejoran al poner en marcha GPC sólo cuando éstas tienen sólida evidencia científica ${ }^{22-24}$. La falta de evidencia que sostiene las recomendaciones ha sido evaluada como uno de los ítems más débiles entre el análisis de las $\mathrm{GPC}^{24}$. Para determinar qué atributos de las GPC influían mayormente en su uso para la toma de decisiones en la práctica médica, Grol et $\mathrm{al}^{7}$, evaluó 12 aspectos (simplicidad, precisión, aplicabilidad, sustento científico, etc) de 47 recomendaciones dadas en una selección de guías clínicas. Al ser implementadas por 61 médicos generales, el 61\% (7.915/12.880) de las decisiones se basaron siguiendo alguna de las recomendaciones de la GPC. Las recomendaciones controversiales fueron aplicadas en sólo el 35\%, contra el 68\% de las no controversiales y $36 \%$ de las vagas contra $67 \%$ de aquellas recomendaciones precisas y concretas. Aquellas recomendaciones que implicaban un cambio importante en la rutina de la práctica clínica fueron menos aplicables (44\%/67\%). Sin embargo, el atributo más importante en el momento de decidir implementar la recomendación, nuevamente fue el peso científico de sus argumentos; así, las recomendaciones basadas en buena evidencia científica fueron mayormente implementadas (71\%/57\%).

Por lo anteriormente expuesto, algún instrumento 
como es el AGREE debiera formar parte del desarrollo y posterior evaluación formal de cualquier GPC, con el objetivo de lograr maximizar el impacto y la aplicabilidad en los profesionales para mejorar el cuidado de los pacientes.

El puntaje final obtenido en la evaluación de esta guía es de un $44,9 \%$, es decir, que cumple con un $44,9 \%$ de los requisitos necesarios para la máxima puntuación posible, lo que en una escala de 1 a 7 equivale a una nota de 3,7 (con punto base). Lejos de desanimarnos, el resultado obtenido pone una nota de alerta para mejorar los puntos peor evaluados y optimizar el alcance y resultados esperables de la incorporación del Gran Quemado al AUGE. Haciendo un diagnóstico inicial de las debilidades, podemos sugerir acciones específicas en los dominios con el fin de mejorar la GCP para su próxima versión:

1) Alcance y objetivo: Definir más específicamente los grupos a tratar en cuanto a edad, superficie quemada y profundidad y sus diferentes relaciones, también el grupo con patología grave asociada. En cuanto a las quemaduras respiratorias: definir si es frente a quemadura sospechada o confirmada y si es confirmada, cuáles son los criterios para su confirmación. También sería necesario aclarar la definición de quemaduras de alta tensión, en cuanto a su profundidad y extensión.

2) Participación de los implicados: Se debe integrar a nuevos profesionales para complementar el manejo integral e interdisciplinario del paciente gran quemado, así como también involucrar a los pacientes y/o a su familia en las necesidades de información respecto al tema y en la toma de decisiones. Es necesario también estudiar las fortalezas y debilidades en la aplicación de las GPC actual y las percepciones de los usuarios y prestadores desde el punto cuantitativo y cualitativo.

3) Rigor en la elaboración: Es necesario detallar la metodología de búsqueda de la evidencia utilizada incluyendo las preguntas a responder. Establecer criterios para seleccionar la evidencia. Ser específico en las recomendaciones y coherente con las referencias obtenidas. Es muy importante una revisión externa y una aplicación piloto para detectar problemas metodológicos o prácticos que ayuden a mejorar en forma eficiente la guía. Se necesita detallar claramente la estrategia de actualización del documento.

4) Claridad y presentación: Se pueden incluir nuevas herramientas como un checklist inicial de las primeras 24 horas, fichas clínicas y de traslado estandarizadas.

5) Aplicabilidad: De suma relevancia es analizar el contexto social, económico y político que influye directamente en las posibilidades reales de ejecución de la GPC. Es importante determinar barreras organizativas como por ejemplo el apoyo logístico para el traslado de paciente y sus familiares, la capacitación del personal, la contratación de recursos humanos necesarios y capacitados para el tratamiento del paciente gran quemado. Es necesaria, la realización de planillas de contabilidad y finanzas en cuanto a costo real de la puesta en marcha de esta GPC del Gran Quemado y los recursos humanos, físicos y financieros reales necesarios para cubrir esta necesidad. Además, generar indicadores de calidad como por ejemplo: presencia de infección en zona dadora de injertos, índice de mortalidad en grupo de paciente crítico y/o sobrevida excepcional, dosis letal media de quemadura (DL50) u otros.

Durante los últimos años, se han publicado avances importantes aplicables a una nueva GPC, destacando revisiones sistemáticas y meta análisis sobre la calidad metodológica de los estudios randomizados realizados en pacientes quemados ${ }^{25}$, manejo tópico de las quemaduras ${ }^{26-29}$, nutrición ${ }^{30,31}$ y rehabilitación ${ }^{32}$; además de GPC acerca de manejo de catástrofes ${ }^{33,34}$, manejo prehospitalario ${ }^{35}$, resucitación $^{36}$, manejo de quemaduras intermedias ${ }^{37}$, criterios diagnósticos de $\operatorname{sepsis}^{38}$, uso de terapia antibiótica $^{39}$, nutrición ${ }^{40,41}$, ventilación mecánica en pacientes quemados ${ }^{42,43}$ y diagnóstico y manejo de neumonía asociada a ventilador mecánico ${ }^{44}$.

Esperamos que nuestro trabajo sea tomado como un aporte al mejoramiento continuo de las GPC más que una crítica a las mismas. Confiamos que el trabajo interdisciplinario de los profesionales que cuidan a los pacientes quemados a lo largo del país, unido a las sociedades científicas, instituciones académicas y organismos de gobierno, culmine en la mejoría de cuidado integral de los pacientes que sufren una de las patologías más devastadoras que le puede ocurrir al ser humano: "Las quemaduras le ocurren a cualquiera, en cualquier lugar y en cualquier momento" 45 .

\section{Referencias}

1. Bastías VG. Reforma de Salud en Chile; El Plan Auge o Régimen de Garantías Explícitas en Salud (Ges). Su Origen y Evolución. Boletín Escuela de Medicina UC, Pontificia Universidad Católica de Chile 2007;2:32.

2. Arrau F. Conceptualización del Plan de Acceso Universal con Garantías Explícitas (AUGE), eje de la actual reforma a la salud. Biblioteca del Congreso Nacional, Serie Estudios de Anticipación 2002; año I(1).

3. Organization WH. Facts about burn injuries. In; 2009.

4. Danilla S, Pasten J, Fasce G, Díaz V, Iruretagoyena M. 
Mortality trends from burn injuries in Chile: 1954-1999. Burns 2004;30:348-56.

5. Chile GD. Guía Clínica Gran Quemado. In: Chile MdSd, ed.; 2007.

6. Grimshaw JM, Russell IT. Effect of clinical guidelines on medical practice: a systematic review of rigorous evaluations. Lancet 1993;342:1317-22.

7. Grol R, Dalhuijsen J, Thomas S, Veld C, Rutten G, Mokkink H. Attributes of clinical guidelines that influence use of guidelines in general practice: observational study. BMJ. 1998;317:858-61.

8. Guyatt GH, Sackett DL, Cook DJ. Users' guides to the medical literature. II. How to use an article about therapy or prevention. A. Are the results of the study valid? Evidence-Based Medicine Working Group. JAMA 1993;270:2598-601

9. Claros C, Vial M, Sanhueza A. Efectividad de la profilaxis antibiótica en el curso de la colecistectomía laparoscópica electiva: revisión sistemática de la literatura. Rev Chil Cir. 2007;59:353-9.

10. Manterola D. Medicina basada en la evidencia o medicina basada en pruebas I: generalidades acerca de su aplicación en la práctica clínica cotidiana. Rev Med Clín Condes 2009;20:125-30.

11. Littlejohns P, Cluzeau F. Guidelines for evaluation. Fam Pract. 2000;17 Suppl 1:S3-6.

12. Cluzeau FA, Littlejohns P, Grimshaw JM, Feder G, Moran SE. Development and application of a generic methodology to assess the quality of clinical guidelines. Int J Qual Health Care 1999;11:21-8.

13. Collaboration TA. AGREE Instrument Spanish Version. In.

14. Field M. Guidelines for Clinical Practice. From development to use. Washington DC National Academy Press 1992.

15. Grilli R, Magrini N, Penna A, Mura G, Liberati A. Practice guidelines developed by specialty societies: the need for a critical appraisal. Lancet 2000;355:103-6.

16. Grimshaw J, Freemantle N, Wallace S. Developing and implementing clinical practice guidelines. Qual Health Care 1995;4:55-64.

17. Shaneyfelt TM, Mayo-Smith MF, Rothwangl J. Are guidelines following guidelines? The methodological quality of clinical practice guidelines in the peer-reviewed medical literature. JAMA 1999;281:1900-5.

18. Grol R, Cluzeau FA, Burgers JS. Clinical practice guidelines: towards better quality guidelines and increased international collaboration. Br J Cancer 2003;89 Suppl $1: S 4-8$.

19. Burgers JS, Grol R, Klazinga NS, Makela M, Zaat J. Towards evidence-based clinical practice: an international survey of 18 clinical guideline programs. Int J Qual Health Care 2003;15:31-45.

20. Grimshaw J, Eccles M, Russell I. Developing clinically valid practice guidelines. J Eval Clin Pract. 1995;1:3748.
21. Worrall G, Chaulk P, Freake D. The effects of clinical practice guidelines on patient outcomes in primary care: a systematic review. CMAJ. 1997;156:1705-12.

22. Bahtsevani C, Uden G, Willman A. Outcomes of evidence-based clinical practice guidelines: a systematic review. Int J Technol Assess Health Care 2004;20:42733.

23. Thomas LH, McColl E, Cullum N, Rousseau N, Soutter $\mathrm{J}$. Clinical guidelines in nursing, midwifery and the therapies: a systematic review. J Adv Nurs. 1999;30:40-50.

24. Wilson MC, Hayward RS, Tunis SR, Bass EB, Guyatt G. Users' guides to the Medical Literature. VIII. How to use clinical practice guidelines. B. what are the recommendations and will they help you in caring for your patients? The Evidence-Based Medicine Working Group. JAMA 1995;274:1630-2.

25. Danilla S, Wasiak J, Searle S, Arriagada C, Pedreros C, Cleland H, Spinks A. Methodological quality of randomised controlled trials in burns care. A systematic review. Burns 2009;35:956-61.

26. Gravante G, Caruso R, Sorge R, Nicoli F, Gentile P, Cervelli V. Nanocrystalline silver: a systematic review of randomized trials conducted on burned patients and an evidence-based assessment of potential advantages over older silver formulations. Ann Plast Surg. 2009;63:201-5.

27. Wasiak J, Cleland H. Topical negative pressure (TNP) for partial thickness burns. Cochrane Database Syst Rev. 2007(3):CD006215.

28. Wasiak J, Cleland H, Campbell F. Dressings for superficial and partial thickness burns. Cochrane Database Syst Rev. 2008(4):CD002106.

29. Wijesinghe M, Weatherall M, Perrin K, Beasley R. Honey in the treatment of burns: a systematic review and metaanalysis of its efficacy. N Z Med J. 2009;122:47-60.

30. Berger MM, Shenkin A. Trace element requirements in critically ill burned patients. J Trace Elem Med Biol. 2007;21 Suppl 1:44-8.

31. Wasiak J, Cleland H, Jeffery R. Early versus late enteral nutritional support in adults with burn injury: a systematic review. J Hum Nutr Diet. 2007;20:75-83.

32. Anzarut A, Olson J, Singh P, Rowe BH, Tredget EE. The effectiveness of pressure garment therapy for the prevention of abnormal scarring after burn injury: a metaanalysis. J Plast Reconstr Aesthet Surg. 2009;62:77-84.

33. Disaster management and the ABA Plan. J Burn Care Rehabil. 2005;26:102-6.

34. Haberal M. Guidelines for dealing with disasters involving large numbers of extensive burns. Burns 2006;32:933-9.

35. Allison K, Porter K. Consensus on the pre-hospital approach to burns patient management. Injury 2004;35:734-8.

36. Pham TN, Cancio LC, Gibran NS. American Burn Association practice guidelines burn shock resuscitation. J Burn Care Res. 2008;29:257-66. 
37. Alsbjorn B, Gilbert P, Hartmann B. Guidelines for the management of partial-thickness burns in a general hospital or community setting-recommendations of a European working party. Burns 2007;33:155-60.

38. Greenhalgh DG, Saffle JR, Holmes JHT. American Burn Association consensus conference to define sepsis and infection in burns. J Burn Care Res. 2007;28:776-90.

39. (SFETB) Sfd,edtdb. [Guidelines for use of antibiotics in burn patient at the acute phase-long text. Societé francaise d'etude et de traitement des brelures]. Ann Fr Anesth Reanim. 2009;28:265-74.

40. Berger MM, Raffoul W, Shenkin A. 'Practical guidelines for nutritional management of burn injury and recovery'-a guideline based on expert opinion but not including RCTs. Burns 2008;34:141-3.

41. Prelack K, Dylewski M, Sheridan RL. Practical gui- delines for nutritional management of burn injury and recovery. Burns 2007;33:14-24.

42. Dries DJ. Key questions in ventilator management of the burn-injured patient (first of two parts). J Burn Care Res. 2009;30:128-38.

43. Dries DJ. Key questions in ventilator management of the burn-injured patient (second of two parts). J Burn Care Res. 2009;30:211-20.

44. Mosier MJ, Pham TN. American Burn Association Practice guidelines for prevention, diagnosis, and treatment of ventilator-associated pneumonia (VAP) in burn patients. J Burn Care Res. 2009;30:910-28.

45. Haberal M. Message from the President. Burns 2007;33:III-IV questions in ventilator management of the burn-injured patient (first of two parts). J Burn Care Res. 2009;30:128-38.

\title{
Anexo 1. Instrumento AGREE (http://www.agreecollaboration.org/)
}

\begin{abstract}
Alcance y objetivo
1. El (Los) objetivo (s) general (es) de la guía está (n) específicamente descrito (s).

2. El (Los) aspecto (s) clínico (s) cubierto (s) por la guía está (n) específicamente descrito (s).

3. Los pacientes a quienes se pretende aplicar la guía están específicamente descritos.
\end{abstract}

\section{Participación de los implicados}

4. El grupo que desarrolla la guía incluye individuos de todos los grupos profesionales relevantes.

5. Se han tenido en cuenta los puntos de vista del paciente y sus preferencias.

Muy de acuerdo $4 \begin{array}{lllll}4 & 2 & 1\end{array}$ Muy en desacuerdo

6. Los usuarios diana de la guía están claramente definidos.

7. La guía ha sido probada entre los usuarios diana.

\section{Rigor en la elaboración}

8. Se han utilizado métodos sistemáticos para la búsqueda de la evidencia.

9. Los criterios para seleccionar la evidencia se describen con claridad.

10. Los métodos utilizados para formular las recomendaciones están claramente descritos.

11. Al formular las recomendaciones han sido considerados los beneficios en salud, los efectos secundarios y los riesgos.

12. Hay una relación explícita entre cada una de las recomendaciones y las evidencias en las que se basan.

13. La guía ha sido revisada por expertos externos antes de su publicación.

14. Se incluye un procedimiento para actualizar la guía.

\section{Claridad y presentación}

15. Las recomendaciones son específicas y no son ambiguas.

16. Las distintas opciones para el manejo de la enfermedad o condición se presentan claramente.

17. Las recomendaciones clave son fácilmente identificables.

18. La guía se apoya con herramientas para su aplicación.

\section{Aplicabilidad}

19. Se han discutido las barreras organizativas potenciales.

20. Han sido considerados los costes potenciales de la aplicación de las recomendaciones.

21. La guía ofrece una relación de criterios clave con el fin de realizar monitorización y/o auditoría.

\section{Independencia editorial}

22. La guía es editorialmente independiente de la entidad financiadora.

23. Se han registrado los conflictos de intereses de los miembros del grupo de desarrollo. 